\title{
Saia Justa: Medindo a Apropriação de Conceitos sobre Planejamento Familiar através de um Jogo Sério para Dispositivos Móveis
}

\author{
Isaias José Ramos de Oliveira ${ }^{1}$ \\ Dimitri Santos ${ }^{1}$ \\ Juliano Gaspar ${ }^{1}$ \\ Zilma Silveira Nogueira Reis ${ }^{1}$ \\ Lorena Carzola ${ }^{2}$ \\ Mário Dias Corrêa-Júnior ${ }^{2}$ \\ ${ }^{1}$ Faculdade de Medicina e Centro de Informática em Saúde da Universidade Federal de Minas Gerais (UFMG), Belo \\ Horizonte, Brasil; E-Mails: isaias@medicina.ufmg.br; dimitribsn@gmail.com; julianogaspar@gmail.com; \\ zilma.medicina@gmail.com; \\ ${ }^{2}$ Faculdade de Medicina da Universidade Federal de Minas Gerais (UFMG), Belo Horizonte, Brasil; E-Mails: \\ Icm_cazorla@hotmail.com; correajr@gmail.com;
}

\begin{abstract}
Resumo
Introdução: A gravidez não desejada continua a ser um desafio global. Recentemente, a m-Health tem oferecido um novo horizonte para a educação em saúde, contribuindo para as políticas públicas de planejamento familiar, já que os dispositivos móveis ocupam lugar de destaque no cotidiano das pessoas. O objetivo deste estudo foi apresentar evidência da apropriação imediata de conceitos sobre planejamento familiar, utilizando-se de estratégia de gamificação, em uma aplicação do tipo jogo sério. Métodologia: A aplicação para dispositivos móveis utilizou tecnologia open-source e frameworks para plataforma híbrida, sendo oferecido gratuitamente em lojas de aplicativos. Como estratégia, o jogo apresentou desesseis situações do cotidiano dos casais acerca de contracepção. Para cada uma, quatro soluções foram preparadas para atender dimensões ligadas à saúde, família, amigos ou pais, apontando tal influência na decisão do jogador. O progresso na aprendizagem de conceitos sobre contracepção foi estimado pela diferença entre o número de métodos contraceptivos conhecidos após e antes do jogo, analisado estatisticamente. Resultados: Em seis meses, o número de downloads foi de 3652 , com 2809 jogadoresadultos, $77 \%$ feminino e $84 \%$ com idade entre 18 e 33 anos. Considerando os países que utilizaram: 39\% foram originários de Moçambique, 33\% do Brasil, $21 \%$ de Angola e $7 \%$ de outros países. Parte dos jogadores adultos $(1070,38 \%)$ completaram os desesseis desafios. Dos 650 jogadores que relataram conhecer algum método contraceptivo previamente, 117 (18\%) apresentaram diferença positiva entre o número de métodos conhecidos após $(p<0,001)$, em relação aos conhedidos antes do jogo. Conclusão: O jogo sério Saia Justa foi capaz de expandir o acesso dos cidadãos à informação sobre métodos contraceptivos e mostrar evidência significativa de apropriação imediata de conceitos, indicando o potencial deste canal de comunicação para apoiar educação em saúde.
\end{abstract}

Palavras-chave: dispositivos móveis; educação em saúde; métodos contraceptivos; planejamento familiar.

\begin{abstract}
Introduction: Unwanted pregnancy remains a global challenge. Recently, m-Health has provided a new horizon for health education as mobile devices occupy a prominente place in people's daily lives. This new perspective contributes to the development of public policies for family planning. The objective of this study was to present evidence of the immediate Appropriation of concepts about family planning, using gamification strategy, in a serious game Application. Methodology: The mobile Application used open-source technology and hybrid platform frameworks, being offered free of charge in App stores. As its strategy, the game presented sixteen situations about
\end{abstract}


contraception in couples' daily lives. For each situation, four solutions were prepared to meet dimensions related to health, family, friends or parents, pointing out their such influence on the player's decision. Progress in learning concepts about contraception was estimated by the difference between the number of contraceptive methods known before and after the game. Results: In six months, the number of downloads was 3652, with 2809 adult players, $77 \%$ female and $84 \%$ with ages between 18 and 33 years. Considering the countries of the user: $39 \%$ were from Mozambique, 33\% from Brazil, $21 \%$ from Angola and $7 \%$ from other countries. Part of the adult players (1070, 38\%) completed all the sixteen challenges. Of the 650 players who reported knowing some contraceptive method previously, 117 (18\%) presented a positive difference between the number of known methods before and after the game, $p<0.001$. Conclusion: The serious game "Saia Justa" was able to expand users access to information on contraceptive methods and to show significant evidence of immediate Appropriation of concepts, indicating the potential of this communication channel to support health education.

Keywords: contraceptive methods; family planning; health education; mobile devices.

\section{Introdução}

A gravidez indesejada permanece um desafio na sociedade atual e a tecnologia da informação pode oferecer soluções inovadoras para dar suporte às políticas públicas de planejamento familiar. As gestações planeadas são aquelas que ocorrem no momento desejado ou a qualquer altura, para as mulheres que são indiferentes ao tempo da gravidez (Logan et al., 2007). Nascimentos não planeados estão associados a resultados neonatais adversos, tais como baixo peso ao nascer, parto prematuro, além das complicações durante a gravidez e para o futuro da criança (Finer \& Kost, 2011). É um dos indicadores mais importantes da saúde reprodutiva de uma população (Finer \& Kost, 2011).

Ajudar as mulheres a planear a gravidez requer uma abordagem abrangente e clara dos riscos reais inerentes a cada método contraceptivo e de se considerar como as mulheres percebem esses riscos sobre si mesmas (Frost, Singh, \& Finer, 2007). Neste sentido, o planejamento familiar é um conjunto de ações e recursos oferecidos para ajudar uma família a ter os filhos que desejar, envolvendo a oferta de métodos contraceptivos e medidas de suporte para livre escolha e sua manutenção (Brasil, 2006). No Brasil, o tal planejamento integra o conjunto de direitos sexuais e reprodutivos, sem a imposição de nenhum método anticoncepcional ou do número de crianças que um cidadão deseja ter (Brasil, 2006).

No entanto, a escolha de um método específico e a motivação para usá-lo ou abandoná-lo é uma questão complexa. Até o momento não há como aferir a intensidade da motivação para o uso de contraceptivos, apenas é assumido que algumas mulheres estão mais interessadas em adotar a contracepção do que outras (Kuang, Ross, \& Madsen, 2014). É especialmente importante o papel dos profissionais de saúde e dos pais para incentivar o uso regular de preservativos. Especialmente a influência das mães sobre as filhas na formação da intenção positiva para o uso do preservativo, de forma correta e no momento certo, assim como o papel do médico nesta orientação (Reis, 2009). As decisões que as mulheres tomam sobre planejamento familiar refletem uma variedade de influências, incluindo informações disponíveis na mídia, acesso a métodos e serviços de saúde, além de influências culturais, religiosas e sociais, padrões socioeconômicos e comunitários (Valdés, Alarcon, \& Munoz, 2013).

A tecnologia móvel tornou-se um potencial a ser explorado na educação de adultos. Acredita-se que sua utilização possa apoiar a educação do cidadão, dada sua presença marcante no cotidiano, independentemente da classe social (Kavanaugh, Lindberg, \& Frost, 2012). Os adultos precisam de métodos de aprendizagem diferentes daqueles usados para crianças, evitando-se que o processo de ensino se torne tedioso e para superar as crenças e inibições negativas prévias (Knowles, 1973). É fato que para eles, a necessidade de conhecimento está diretamente ligada à sua aplicação na solução de problemas cotidianos (Knowles, 1973). Adultos são motivados internamente e auto-dirigidos para aprender, mas trazem experiências de vida que devem ser respeitadas (Lichtenstein, 2016).

Os jogos são atividades com valor educacional intrínsecos, capazes de estimular a atenção, enquanto motivam o jogador através de competição e cooperação. Os jogos educativos proporcionam uma contribuição relevante para o aprendizado e para disseminar informações (Calisto, Barbosa, \& Silva, 2010). Jogos sérios têm sido empregados para treinamento em educação, ciência e nas atividades laborais (Derryberry, 2007). Eles são intencionalmente feitos para aprender, uma vez que têm grande potencial para suportar experiências de aprendizagem imersivas, um passo crucial para a aprendizagem (Bizzocchi \& Paras, 2005). As aplicações (App) para dispositivos móveis vêm criando novas 
oportunidades de alcançar populações, que eram em grande parte inacessíveis através de canais de cuidados de saúde tradicionais (Fischer, 2001).

Dada a questão desafiadora da gravidez indesejada em nossa sociedade, o alcance da tecnologia móvel carregando a estratégia de um jogo sério, o estudo teve como objetivo avaliar o impacto de uma App sobre planejamento familiar na disseminação de conceitos sobre o tema.

\section{Metodologia}

Trata-se de um estudo interdisciplinar entre as ciências da saúde e da computação, de natureza exploratória e aplicada, apresentando o desenvolvimento, disseminação e retorno dos utilizadores sobre uma App para dispositivos móveis. Do ponto de vista epidemiológico foi um estudo de seguimento de coorte prospectiva, dado que acompanhou-se o desempenho dos jogadores antes e após sua utilização. O estudo foi aprovado no Comitê de Ética e Pesquisa da UFMG sob o número C.A.A.E. 48435815.2.0000.5149.

A abordagem científica decorreu-se em etapas: a primeira foi a revisão de literatura científica sobre o tema planejamento familiar, com foco nas melhores práticas em saúde. Em seguida, a estratégia do jogo sério foi elaborada partindo-se de desafios contendo situações-problema, associados ao cotidiano de casais heterosexuais. Tais desafios foram validados quanto a relevância linguagem utilizada nos textos, por usuários voluntários, valendo-se de grupos focais coordenados por médicos ginecologistas. Uma lista de situações fictícias previamente preparadas, com formato voltado predominantemente para o público feminino, buscou abranger contextos diversos de forma a permitir a introdução de conteúdos relativos aos métodos contraceptivos disponíveis no país, assim como estratégias para as tomadas de decisão em conjunto, pelo casal, ou individual. Além disto, todo conteúdo técnico-científico foi revisado por especialistas, professores da Faculdade de Medicina da Universidade Federal de Minas Gerais.

Como estratégia, o jogo apresentou dezesseis situações do cotidiano dos casais acerca de contracepção (Figura 1). Para cada uma, quatro soluções foram preparadas para atender dimensões ligadas à saúde, família, amigos ou pais, apontando tal influência na decisão do jogador. Para cada decisão tomada, um retorno foi apresentado, a partir da implementação de um personagem (avatar) chamado "Doutora Sabe-Mais", cujo papel foi de reforço positivo, apontamento de riscos associados às decisões e de oferecer conteúdo adicional com base científica sobre a questão. Procedeu-se então a etapa de programação do App para dispositivos móveis, por equipe do Centro de Informática em Saúde da Faculdade de Medicina da UFMG. Para isto, utilizou-se tecnologia open-source e frameworks para plataforma híbrida, com ilustrações produzidas por profissional especialista. 


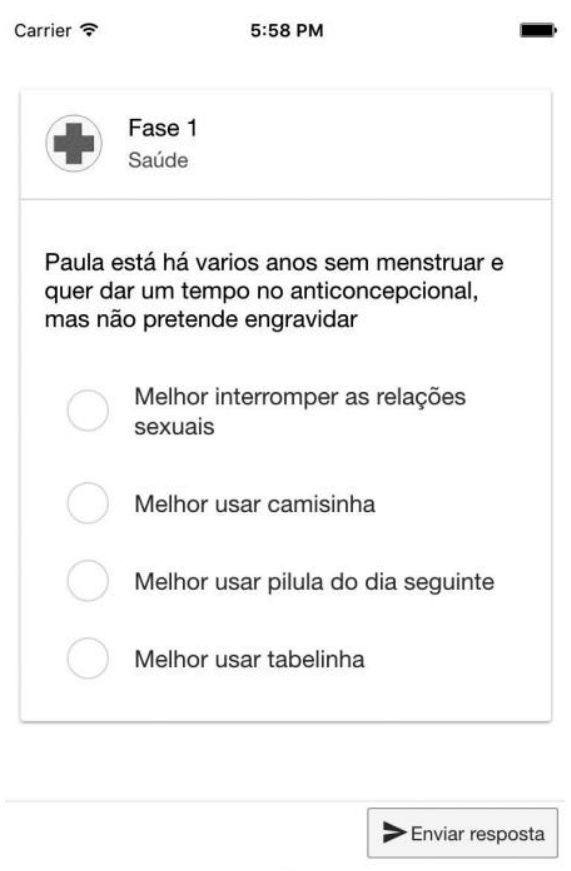

(A)

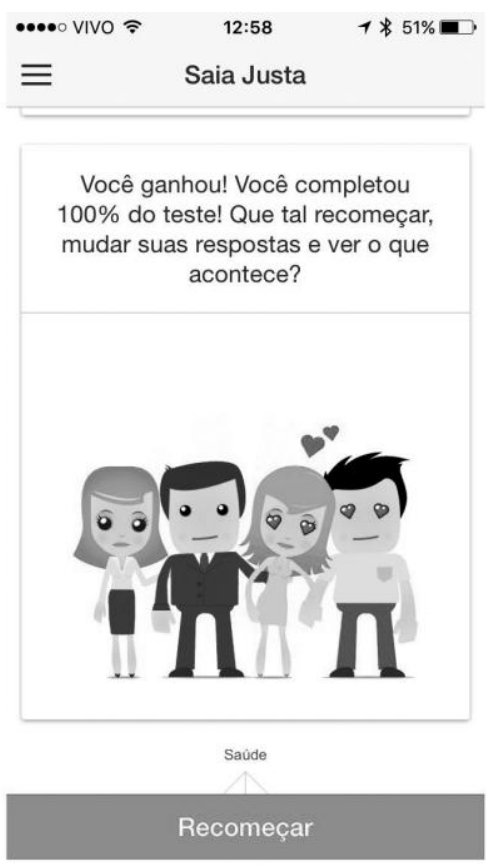

(B)

Figura 1. Exemplo de interfaces da App Saia Justa: (A) Desafio sobre contracepção e as respectivas opções de decisões para o jogador. (B) Mensagem de finalização do jogo no aplicativo Saia Justa.

O jogo sério foi então disponibilizado gratuitamente em lojas de App na internet, em Abril de 2016, com a recomendação para uso por adultos e acompanhada do termo de consentimento on line explicitando fins de pesquisa. Na última etapa do estudo, após seis meses de lançamento do App e livre utilização pelo público interessado, os dados recolhidos sobre os utilizadores foram utilizados para análise estatística. 0 progresso na apropriação de conceitos sobre contracepção foi estimado pela diferença entre o número de métodos contraceptivos conhecidos antes e após do jogo. A análise foi realizada através do teste pareado, não paramétrico, de Wilcoxon. Empregou-se o software SPSS versão 22 IBM, considerando-se significativa a probabilidade de significância menor que 5\%.

\section{Resultados}

O jogo sério denominado Saia Justa foi baixado através da lojas de App por 3.652 utilizadores. Estima-se que o jogo tenha sido utilizado por 3070 jogadores, cerca de 1,38 vezes por dispositivo móvel. Quanto ao sistema operacional do dispositivo móvel, o sistema iOS respondeu por 248 (6,8\%) downloads contra 3.404 (93\%) dispositivos com sistema Android. Relativamente ao país de origem do utilizador do App, 39\% tem origem em Moçambique, 33\% no Brasil, 21\% emAngola, Cabo Verde $4 \%$ e $3 \%$ em outros países.

O tempo médio de jogo foi de 7:33 minutos. Entre os adultos que informaram a idade, 1462 pessoas (52\%), a faixa variou de 18 a 77 anos, com maior frequência entre adultos jovens entre 18 e 33 anos, grupo que totalizou 1.233 (84\%) dos jogadores que informaram a idade. No entanto, além de aceder ao App, foram 1.070 os que completaram todo o percurso do jogo pelo menos uma vez, sendo selecionados para análise, à partir da última utilização registrada na data deste estudo. Na Tabela 1, os utilizadores do App selecionados foram caracterizados quanto à auto-avaliação sobre o conhecimento prévio ao jogo, em relação aos métodos contraceptivos. Nota-se que a maioria julgava de antemão já ter bom ou elevado conhecimento prévio sobre as técnicas de planejamento familiar. 
Tabela 1. Conhecimento prévio sobre métodos contraceptivos que o utilizador alegou saber antes de jogar.

\begin{tabular}{cc}
\hline Nível de conhecimento & $\mathbf{n ~ ( \% )}$ \\
\hline Alto & $126(11,8)$ \\
Bom & $410(38,3)$ \\
Baixo & $143(13,4)$ \\
Não informado & $391(36,5)$ \\
Total & $1070(100,0)$ \\
\hline
\end{tabular}

Especificamente quanto à aprendizagem, dentre 650 jogadores, observou-se que houve uma diferença significativa entre o número de métodos contraceptivos que o jogador relatou conhecer ao final do jogo, em relação ao que já conhecia previamente $(p<0,001)$, Tabela 2. Parte desta diferença foi positiva, em 117 (18\%) dos jogadores, ou seja, o número de métodos relatados após o jogo foi maior que o prévio, evidenciando retenção significativa de novos conceitos sobre o tema.

Tabela 2. Diferença entre o número de métodos contraceptivos informados pelo jogador no final do jogo em comparação com o número relatado anteriormente

\begin{tabular}{llll}
\hline & $\mathbf{n}$ & $\%$ & $p$-value \\
\hline Diferença positiva & 117 & 18,0 & $<0,001$ \\
Diferença negativa & 10 & 1,5 & \\
Empates & 523 & 80,5 & \\
\hline
\end{tabular}

* Wilcoxon test

\section{Discussão}

No universo da informação disponível para o jovem adulto contemporâneo, imerso em ambiente virtual, considera-se oportuno que se desenvolvam novas possibilidades de aconselhamento contraceptivo por esse meio de comunicação. Dadas as relevantes complicações associadas à gravidez não planeada e o grande volume de informação na internet, torna-se ainda maior a importância do acesso democrático às fontes confiáveis de informação sobre o assunto. Os resultados aqui apresentados trouxeram a experiência de uma abordagem em formato de jogo sério, na expectativa de que tal estratégia possa apoiar a disseminação de boas práticas em saúde reprodutiva. Evidenciou-se que a intervenção educativa, mediada pela tecnologia da informação, pode representar um ganho para a abordagem populacional. Desta forma, este estudo ofereceu uma contribuição relevante ao retratar a experiência de ensinar saúde para adultos, através de um App previamente preparado e validado em ambiente acadêmico. Destaca-se por avaliar o impacto na aquisição de conhecimento sobre saúde reprodutiva de seus utilizadores.

A estratégia de apresentação progressiva dos conteúdos sobre o tema planejamento familiar foi a de manter uma relação estreita com a construção do conhecimento, através de situações simuladas, onde o jogador se coloca no lugar do personagem e toma decisões. Com isto, acredita-se que o App possa influenciar e motivar o processo de ensino e aprendizagem, envolvendo pelo menos memorização imediata. Há estudos que indicam que a memória humana absorve cerca de $90 \%$ das novas informações através da ação e, portanto, o jogo também pode ser um instrumento capaz de fomentar a memorização, contextualizada em uma situação lúdica e com base na solução de problemas do cotidiano (Benítez, 2010). O jogo aborda, de forma inédita em ambiente virtual de aprendizagem, assuntos relacionados ao uso de contraceptivos, posicionando o jogador em situações desafiadoras para tomada de decisão. Esta estratégia foi concebida pelos autores deste estudo a partir dos pilares da andragogia, visto que a resolução de problemas incentiva o raciocínio do adulto em relação ao conhecimento anterior e estimula o interesse pelo conteúdo (Matai \& Matai, 2009; Knowles, 1973).

Tais situações simuladas contextualizadas no período de vida das pessoas que buscam orientações contraceptivas, passou por ajustes em grupos de planejamento familiar dentro da universidade, para se obter um formato de fácil entendimento. As situações no jogo tentam imitar o círculo de amigos, autocuidado e influência do parceiro e família nas decisões, sem necessariamente focar em respostas totalmente certas ou erradas. 
Para cada decisão tomada, um retorno foi oferecido reforçando conceitos ou alinhando-os para melhor prática em saúde pelo personagem "Doutora Sabe- -Mais". A informação sobre o uso correto dos métodos anticoncepcionais é desejável e estratégica, dada a relação existente entre o conhecimento dos métodos e os comportamentos da contracepção (Wannmacher, 2005). O comportamento sexual de risco e as inequidades em saúde sexual podem ser ainda maiores entre os jovens vulneráveis e para as minorias étnicas (Straw \& Porter, 2012). Sob este aspecto, os dispositivos móveis podem atingir populações que anteriormente tinham acesso restrito a informações ou informações de saúde (Pratt et al., 2012). O jogo tem o potencial de abrir novos caminhos no estabelecimento de uma comunicação agradável com o público, considerando o papel de suas experiências pessoais.

App para dispositivos móveis são softwares desenvolvidos com uma interface simplificada em relação aos computadores e utilizam um desenho e tecnologia para prorporcionar menor tempo dispendido pelo utilizador, facilitando a interatividade, o que melhora a retenção das informações (Zhao, Freeman, \& Li, 2016). Existem aproximadamente 1,8 milhão de aplicativos no Google Play, sendo cerca de 84 mil com fins educacionais, mas nenhum sobre sexualidade ou contracepção. Já na loja de App Apple Store, entre dois milhões de aplicativos disponíveis, mais de 600 mil são jogos e a quantidade de jogos educativos é ainda maior do que no Google Play, pois encontram-se cerca de 2000 App educacionais, gratuitos e disponíveis em português (AppAnnie, 2016). No entanto, nenhuma delas aborda educação sexual em idioma português. Mas é importante destacar que outras experiências neste sentido foram documentadas em inglês. Um grupo de pesquisadores noruegueses desenvolveram um App para promover a educação sexual através de um jogo (Gabarron et al., 2012). Este jogo, apesar de estar online, não está disponível para dispositivos móveis e está focado apenas em jovens daquele país.

Os resultados aqui apresentados devem entretanto ser considerados com cautela. A experiência de se analisar dados obtidos à distância e sem interação direta com os sujeitos da pesquisa é muito recente. Constatou-se uma perda significativa de participantes entre os 3.652 utilizadores que acederam ao App e os 1070 que efetivamente foram incluídos na análise, por informarem ser adultos e terem completado todo o percurso dos dezesseis desafios do jogo sério. Tomou-se como verdadeira a premissa de que os dados inseridos pelo utilizador são reais. Nota-se que nesta seleção, a maioria já tinha bom ou ótimo conhecimento prévio sobre métodos contraceptivos. Mesmo assim, a análise mostrou evidência estatística de que é possível acrescentar informações novas sobre outros métodos e contextos para sua utilização.

Conclui-se que a pesquisa aqui descrita trouxe uma nova abordagem sobre um conteúdo educacional em saúde no formato de jogo, na perpectiva de que tal estratégia possa apoiar a disseminação de boas práticas em saúde reprodutiva. Com foco na prevenção de gravidez não planeada, o emprego de meios da tecnologia da informação mostrou-se efetivo como estratégia de abordagem populacional de apoio à educação para saúde.

\section{Referências}

AppAnnie. (2016). App Annie 2015 Retrospective: Monetization Opens New Frontiers. Accessed 18/10/2016. Retrieved from https://www.Appannie.com/insights/market-data/App-annie-2015-retrospective/.

Benítez, G. S. (2010). Las estrategias de aprendizaje a través del componente lúdico. MarcoELE: Revista de Didáctica Español Lengua Extranjera, (11),20.

Bizzocchi, J., \& Brad P. (2005). Game, motivation, and effective learning: An integrated model for educational game design. Proceedings of DiGRA 2005 Conference.

Brasil. (2006). Direitos sexuais, direitos reprodutivos e métodos anticoncepcionais. edited by Ministério da Saúde. Brasilia: Secretaria de Atenção à Saúde.

André, C., Barbosa, D., \& Silva, C. (2010). Uma análise comparativa entre jogos educativos visando a criação de um jogo para educação ambiental. Anais do Simpósio Brasileiro de Informática na Educação.

Derryberry, A. (2007). Serious games: online games for learning. Adobe White Paper.

Finer, L. B., \& Kost, K. (2011). Unintended pregnancy rates at the state level. Perspectives on Sexual and Reproductive Health, 43(2), 78-87. 
Fischer, G. (2001). User modeling in human-computer interaction. User modeling and user-adapted interaction, 11(1), 65-86.

Frost, J. J., Singh, S. \& Finer, L. (2007). Factors associated with contraceptive use and nonuse, United States, 2004. Perspectives on sexual and reproductive health, 39(2), 90-99.

Gabarron, E., Serrano, A., Wynn, R., \& Armayones, M. (2012). Avatars using computer/smartphone mediated communication and social networking in prevention of sexually transmitted diseases among North-orwegian youngsters. BMC medical informatics and decision making, 12(1), 1.

Kavanaugh, M. L., Lindberg, L. \& Frost, J. (2012). Factors influencing partners' involvement in women's contraceptive services. Contraception, 85(1), 83-90.

Knowles, M. (1973). The adult learner: a neglected species.

Kuang, B., Ross, J. \& Madsen, E. (2014). Defining motivational intensity of need for family planning in Africa. African journal of reproductive health, 18(3), 57-66.

Lichtenstein, N. (2016). A história da andragogia, accessed 08/11/2016. Retrieved from http://www.ehow.com.br/historia-andragogia-info_41783/.

Logan, C. E., J. Manlove, \& Ryan, S. (2007). The consequences of unintended childbearing: A white paper. Washington: Child Trends.

Matai, L., \& Matai, S. (2009). Cooperative education: Andragogy. ICEE 2007: Proceedings Coimbra-Universidade de Coimbra.

Pratt, M., Sarmiento, O. L., Montes, F., Marcus, O., Perez, L., \& Brownson, R. (2012). The implications of megatrends in information and communication technology and transportation for changes in global physical activity. Lancet 380 (9838), 282-93. doi: 10.1016/ s0140-6736(12)60736-3.

Reis, Z. (2009). Intenção de uso de preservativo masculino entre jovens estudantes de Belo Horizonte: um alerta aos ginecologistas. Rev Bras Ginecol Obstet, 31(11), 574-80.

Straw, F. \& Porter, C. (2012). Sexual health and contraception. Archives of disease in childhood-Education \& practice edition, 97(5), 177-184.

Valdés, P. R., Alarcon, A., \& Munoz, S. (2013). Evaluation of Informed Choice for contraceptive methods among women attending a family planning program: conceptual development; a case study in Chile. Journal of clinical epidemiology, 66(3), 302-307.

Wannmacher, L. (2005). Contracepção de emergência: evidências versus preconceitos. Uso Racional de Medicamentos: Temas Selecionados 2(6).

Zhao, J., Freeman, B. \& Li, M. (2016). Can mobile phone Apps influence people's health behavior change? An evidence review. Journal of Medical Internet Research, 18(11), e287.

\section{Sobre os autores}

Isaias José Ramos de Oliveira (MsC) possui graduação em Ciência da Computação pela Universidade Federal de Minas Gerais (2010) e mestrado em Medicina (Obstetrícia e Ginecologia) pela Universidade Federal de Minas Gerais (2016). Atualmente é sócio - Butec Inovação llimitada e técnico em tecnologia da informação da Universidade Federal de Minas Gerais. Tem experiência na área de Ciência da Computação, com ênfase em Ciência da Computação, atuando principalmente nos seguintes temas: atividades educacionais, educação em saúde, métodos contraceptivos, planejamento familiar e aplicativos móveis.

Dimitri Bassani Santos Neves possui graduação em Desenho Industrial / Programação Visual pela UFES - Universidade Federal do Espírito Santo (2006). Desde 2013 atuando como web designer terceirizado no Centro de Informática em Saúde (CINS) da Faculdade de Medicina da UFMG e instrutor de cursos de informática na modalidade extensão desta mesma Faculdade. Proprietário da Weiss Code \& Design (MEI) com atuação no ramo editorial, identidade institucional, web e vídeo design, divulgação impressa e digital.

Juliano de Souza Gaspar (PhD) leciona as disciplinas de Bioestatística, Epidemiologia, Sistemas de Informação em Saúde e Informática Médica. Desde 2009 é membro do CINTESIS (Centro de Investigação em Tecnologias e Sistemas de Informação em Saúde da Faculdade de Medicina da Universidade do Porto, Portugal), onde realiza atividades de pesquisador científico. Doutorado em Informática Médica pelo programa de Pós- 
Graduação da Saúde da Mulher da Faculdade de Medicina da UFMG. Concluiu o Mestrado em Informática Médica pela Faculdade de Medicina da Universidade do Porto (2011), após obter o Bacharelato em Ciências da Computação pela Universidade do Vale do Itajaí (2006). É membro do núcleo de Informática em Saúde da Faculdade de Medicina da UFMG, onde desenvolve atividade de investigação científica na área da saúde e a informática, participa como educador nos grupos de iniciação científica e pesquisa, bem como na elaboração e publicação de artigos. Trabalhou 13 anos em empresas privadas na área de informática, suas principais atividades foram como Analista de Sistemas e Coordenação de Projetos, tendo também atuado como instrutor de informática e designer gráfico.

Lorena Carvalho Maia Carzola pertence à Faculdade de Medicina da Universidade Federal de Minas Gerais, Belo Horizonte, Brasil. Possui ensino medio (segundo grau) pelo Colégio Visão (2011). Tem experiência na área de Medicina.

Mário Dias Corrêa-Júnior atualmente é Professor Adjunto da Faculdade de Medicina da UFMG e Coordenador Clínico da Maternidade do Hospital das Clínicas. Atua principalmente na área de Obstetrícia, com ênfase em gravidez de alto risco. Doutorado em Obstetrícia pela UFMG (2006). Mestrado em Obstetrícia pela UFMG (2002). Graduado em Medicina pela Universidade Federal de Minas Gerais (1997), residência em Ginecologia e Obstetrícia pela Santa Casa de Belo Horizonte (2000).

Zilma Silveira Nogueira Reis atualmente é Professora Associada do Departamento de Ginecologia e Obstetrícia, do Programa de Pósgraduação em Saúde da Mulher da UFMG e Médica do Hospital das Clínicas. Coordena o Centro de Informática Médica da Faculdade de Medicina da UFMG desde 2012. É Vice-presidente da Sociedade Brasileira de Informática em Saúde (SBIS). Doutora em Medicina (2002) pela Universidade Federal de Minas Gerais, quando obteve bolsa de doutorado-sanduiche na Alemanha, PROBRAL 113/00 (CAPES/DAAD). Realizou Estágio Sênior PósDoutoral no Exterior com bolsa CAPES, na Universidade do Porto, Portugal (2011). Mestre em Medicina (1993), Médica ginecologista e obstetra (1990). 